Research Article

\title{
Existence Theorem for Noninstantaneous Impulsive Evolution Equations
}

\author{
Gul I Hina Aslam (D), ${ }^{1}$ Amjad Ali, ${ }^{2}$ and Maimona Rafiq ${ }^{3}$ \\ ${ }^{1}$ Pakistan Navy Engineering College, National University of Sciences and Technology, Karachi, Pakistan \\ ${ }^{2}$ Faculty of Science and Technology, University of Debrecen, Debrecen, Hungary \\ ${ }^{3}$ Department of Mathematics, Comsats University Islamabad, Attock 43600, Pakistan \\ Correspondence should be addressed to Gul I Hina Aslam; gul.hina@pnec.nust.edu.pk
}

Received 24 February 2021; Accepted 13 July 2021; Published 26 July 2021

Academic Editor: Ali Jaballah

Copyright () 2021 Gul I Hina Aslam et al. This is an open access article distributed under the Creative Commons Attribution License, which permits unrestricted use, distribution, and reproduction in any medium, provided the original work is properly cited.

In this note, the variational form of the classical Lax-Milgram theorem is used for the divulgence of variational structure of the first-order noninstantaneous impulsive linear evolution equation. The existence and uniqueness of the weak solution of the problem is obtained. In future, this constructive theory can be used for the corresponding semilinear problems.

\section{Introduction}

Evolution equations interpret the differential law of development with respect to time. A vast theory, in this regard, has been developed [1-9]. In [10], Hernándaz and O’Regan established the theory of noninstantaneous impulsive equations and showed the existence of corresponding mild solutions. Recently, in [11], JinRong Wang acquired ample conditions to guarantee the asymptotic stability of linear and semilinear noninstantaneous impulsive evolution equations. In [12], Tang and Nieto used the variational method to show existence of the solution to impulsive evolution equations. However, this approach, to the best of our knowledge, has not been used to show the existence (uniqueness) of the solution to noninstantaneous impulsive evolution equations.

In this paper, we intend to acquire the variational structure associated to the following first-order noninstantaneous impulsive linear evolution equations:

$$
\left\{\begin{array}{l}
u^{\prime}(t)=A u(t), \quad t \in\left[s_{i}, t_{i+1}\right], i \in \mathbb{N}_{0}:=\{0,1,2, \ldots\} \\
u\left(t_{i}^{+}\right)=\left(I+B_{i}\right) u\left(t_{i}^{-}\right), \quad i \in \mathbb{N}:=\{1,2, \ldots\} \\
u(t)=\left(I+B_{i}\right) u\left(t_{i}^{-}\right), \quad t \in\left(t_{i}, s_{i}\right], i \in \mathbb{N} \\
u\left(s_{i}^{+}\right)=u\left(s_{i}^{-}\right), \quad i \in \mathbb{N},
\end{array}\right.
$$

where $A: D(A) \subseteq V \longrightarrow V$ is the infinitesimal generator of a strongly continuous semigroup of linear operators $\{Z(t)\}_{t \geq 0}$ on a Banach space $V$ endowed with a norm, the impulsive jump operator $B_{i} \in B(V), i \in \mathbb{N}$, and the sequences $\left\{t_{i}\right\}_{i \in \mathbb{N}_{0}}$ and $\left\{s_{i}\right\}_{i \in \mathbb{N}_{0}}$ satisfy the relation $t_{i}<s_{i}<t_{i+1}, i \in \mathbb{N}_{0}$ and set $t_{0}=s_{0}=0$. Furthermore, $I$ denotes the identity operator.

\section{Preliminaries}

Let $\Omega=(0, \tau) \subset \mathbb{R}$ be an open set and let $1 \leq p \leq \infty$. The Sobolev space $W^{1, p}(\Omega)$ is the space of all functions $u \in L^{p}(\Omega)$ whose distributional first-order derivatives 
belong to $L^{p}(\Omega)$; that is, there exists a function $v \in L^{p}(\Omega)$ such that

$$
\int_{\Omega} u(t) \phi^{\prime} \mathrm{d} t=-\int_{\Omega} v(t) \phi(t) \mathrm{d} t,
$$

for all $\phi \in C_{c}^{\infty}(\Omega)$. The function $\nu$ is called the weak or distributional derivative of $u$. The space $W^{1, p}(\Omega)$ is a Banach space with respect to the following norm:

$$
\|u\|_{W^{1, p}(\Omega)}:=\|u\|_{L^{p}(\Omega)}^{p}+\left\|u^{\prime}\right\|_{L^{p}(\Omega)}^{p} .
$$

The space $W_{0}^{1, p}(\Omega)$ is defined as the closure of the space $C_{c}^{\infty}(\Omega)$ in $W^{1, p}(\Omega)$ (with respect to the topology of $\left.W^{1, p}(\Omega)\right)$. Precisely, this space is

$$
W_{0}^{1, p}(\Omega):=\left\{u \in W^{1, p}(\Omega):\left.u\right|_{\partial \Omega}=0\right\} .
$$

A special case is when $p=2$. Then, it is often written as $H_{0}^{1}(\Omega)$ which is a Hilbert space with the following inner product:

$$
(u, v)=\int_{0}^{\tau} u(t) v(t) \mathrm{d} t
$$

where the corresponding norm is

$$
\|u\|=\left(\int_{0}^{\tau}|u(t)|^{2} \mathrm{~d} t\right)^{1 / 2} .
$$

First, let us review that, for

$$
\|u\|_{\infty}=\sup _{t \in[0, \tau]}|u(t)|,\|u\|_{L^{2}}:=\left(\int_{0}^{\tau}|u(t)|^{2} \mathrm{~d} t\right)^{1 / 2},
$$

we have, for $u \in H_{0}^{1}(0, \tau)$,

$$
\begin{aligned}
& \|u\|_{L^{2}} \leq \frac{\tau}{\pi}\left\|u^{\prime}(t)\right\|_{L^{2}}, \\
& \|u\|_{\infty} \leq \alpha\|u\|,
\end{aligned}
$$

where $\alpha=\pi(\tau)^{-1 / 2}+\tau^{1 / 2}$.

Definition 1. Let $X$ and $Y$ be Banach spaces. $\mathscr{B}: X \times Y \longrightarrow \mathbb{R}$ is nondegenerate with respect to the second variable if, for each $y \in Y /\{0\}$, there exists $x \in X$ such that $\mathscr{B}(x, y) \neq 0$.

In [13], Drivaliaris and Yannakakis proved the following variant form of generalized Lax-Milgram Theorem [14].

Theorem 1. Suppose $X$ and $Y$ are Banach Spaces, $X$ is reflexive, and that $\mathscr{B}: X \times Y \longrightarrow \mathbb{R}$ is a bounded, nondegenerate (w.r.t the second variable), and bilinear functional. There exists an $m>0$ such that, for each $x \in X$, $\sup _{\|y\|=1}|\mathscr{B}(x, y)| \geq m\|x\|$.

Then, for every bounded linear functional $S$ on $Y^{*}$, there exists a unique $x \in X$ with

$$
\mathscr{B}(x, y)=S(y),
$$

for all $y \in Y$.

\section{Main Results}

Using the technique of variational approach to impulsive differential equations of $[15,16]$, we have following results.

Lemma 1. $A: D(A) \subseteq V \longrightarrow V$ being the infinitesimal generator of a strongly continuous semigroup of linear operators $\{Z(t)\}_{t \geq 0}$ on a Banach space $V$ endowed with a norm. For each $v \in H_{0}^{1}(0, \tau)$, problem (1) has the following equivalent form:

$$
\int_{0}^{\tau} u^{\prime}(t) v(t) \mathrm{d} t=\sum_{i=1}^{N}\left[B_{i} Z\left(t_{i}\right) v\left(t_{i}\right)\right]+\sum_{i=0}^{N} \int_{s_{i}}^{t_{i+1}} A s_{i} Z(t) v(t) \mathrm{d} t .
$$

Proof

$$
\begin{aligned}
\int_{0}^{\infty} u^{\prime}(t) v(t) \mathrm{d} t & =\int_{0}^{t_{1}} u^{\prime}(t) v(t) \mathrm{d} t+\sum_{i=1}^{N} \int_{t_{i}}^{s_{i}} u^{\prime}(t) v(t) \mathrm{d} t+\sum_{i=1}^{N-1} \int_{s_{i}}^{t_{i+1}} u^{\prime}(t) v(t) \mathrm{d} t+\int_{s_{N}}^{\tau} u^{\prime}(t) v(t) \mathrm{d} t+\int_{\tau}^{\infty} u^{\prime}(t) v(t) \mathrm{d} t \\
& =-\int_{0}^{\tau} u(t) v^{\prime}(t) \mathrm{d} t+\sum_{i=1}^{N} u\left(s_{i}^{-}\right) v\left(s_{i}\right)-\sum_{i=1}^{N} u\left(s_{i}^{+}\right) v\left(s_{i}\right)+\sum_{i=1}^{N} u\left(t_{i}^{-}\right) v\left(t_{i}\right)-\sum_{i=1}^{N} u\left(t_{i}^{+}\right) v\left(t_{i}\right)
\end{aligned}
$$

or

$$
\int_{0}^{\infty} u^{\prime}(t) v(t) \mathrm{d} t=\sum_{i=1}^{N}\left[u\left(t_{i}^{-}\right)-u\left(t_{i}^{+}\right)\right] v\left(t_{i}\right)-\int_{0}^{\tau} u(t) v^{\prime}(t) \mathrm{d} t .
$$

Using the conditions of (1),

$$
\int_{0}^{\infty} u^{\prime}(t) v(t) \mathrm{d} t=-\sum_{i=1}^{N} B_{i} u\left(t_{i}^{-}\right) v\left(t_{i}\right)-\int_{0}^{\tau} u(t) v^{\prime}(t) \mathrm{d} t .
$$


While, on the contrary,

$$
\begin{aligned}
\int_{0}^{\infty} u^{\prime}(t) v(t) \mathrm{d} t & =\sum_{i=0}^{N} \int_{s_{i}}^{t_{i+1}} u^{\prime}(t) v(t) \mathrm{d} t+\sum_{i=1}^{N} \int_{t_{i}}^{s_{i}} u^{\prime}(t) v(t) \mathrm{d} t+\int_{\tau}^{\infty} u^{\prime}(t) v(t) \mathrm{d} t \\
\sum_{i=0}^{N} \int_{s_{i}}^{t_{i+1}} u^{\prime}(t) v(t) \mathrm{d} t & =\sum_{i=0}^{N} \int_{s_{i}}^{t_{i+1}} A u(t)(t) v(t) \mathrm{d} t \\
\sum_{i=1}^{N} \int_{t_{i}}^{s_{i}} u^{\prime}(t) v(t) \mathrm{d} t & =0
\end{aligned}
$$

Therefore,

$$
\int_{0}^{\infty} u^{\prime}(t) v(t) \mathrm{d} t=\sum_{i=0}^{N} \int_{s_{i}}^{t_{i+1}} A u(t) v(t) \mathrm{d} t
$$

Comparing (14) and (16) yields

$$
\int_{0}^{\tau} u(t) v^{\prime}(t) \mathrm{d} t=-\left[\sum_{i=1}^{N}\left[B_{i} u\left(t_{i}^{-}\right) v\left(t_{i}\right)\right]+\sum_{i=0}^{N} \int_{s_{i}}^{t_{i+1}} A u(t) v(t) \mathrm{d} t\right] .
$$

Since, for $t \in\left[s_{i}, t_{i+1}\right], u^{\prime}(t)=A u(t)$, where $A$ is an infinitesimal generator of a $C_{0}$-semigroups $\{Z(t)\}_{t \geq 0}$, therefore, $u(t)=s_{i} Z(t)$, for $t \in\left[s_{i}, t_{i+1}\right]$. For details, we refer $[3,17]$. Hence, (17) becomes

$$
\int_{0}^{\tau} u(t) v^{\prime}(t) \mathrm{d} t=-\left[\sum_{i=1}^{N}\left[B_{i} Z\left(t_{i}\right) v\left(t_{i}\right)\right]+\sum_{i=0}^{N} \int_{s_{i}}^{t_{i+1}} A s_{i} Z(t) v(t) \mathrm{d} t\right]
$$

or

$$
\int_{0}^{\tau} u^{\prime}(t) v(t) \mathrm{d} t=\sum_{i=1}^{N}\left[B_{i} Z\left(t_{i}\right) v\left(t_{i}\right)\right]+\sum_{i=0}^{N} \int_{s_{i}}^{t_{i+1}} A s_{i} Z(t) v(t) \mathrm{d} t
$$

Lemma 2. For a bilinear form $\mathscr{B}(u, v)=\int_{0}^{\tau} u(t) v(t) d t$ on $H_{0}^{1}(0, \tau)$, there exists a $k>0$ such that, for each $u \in H_{0}^{1}(0, \tau)$, $\sup _{\|v\|=1}|\mathscr{B}(u, v)| \geq k\|u\|$.

Proof. Since a bounded bilinear form $\mathscr{B}(u, v)$ on a Hilbert space $H$ is of the form $(F u, v)$, for a unique bounded operator $F$ on $H$, in this case, if we choose $F=u^{\prime}$, we have

$$
(F u, v)=\int_{0}^{\tau} u^{\prime}(t) v(t) \mathrm{d} t=\mathscr{B}(u, v) .
$$

By using (8), the result follows.

Define $S: H_{0}^{1}(0, \tau) \longrightarrow \mathbb{R}:$

$$
S(v)=\sum_{i=1}^{N}\left[B_{i} Z\left(t_{i}\right) v\left(t_{i}\right)\right]+\sum_{i=0}^{N} \int_{s_{i}}^{t_{i+1}} A s_{i} Z(t) v(t) \mathrm{d} t
$$

Note that a function $u \in H_{0}^{1}(0, \tau)$ is the weak solution of problem (1) if and only if

$$
\mathscr{B}(u, v)=S(v), \quad \forall v \in H_{0}^{1}(0, \tau) .
$$

It can be easily noted that $H_{0}^{1}(0, \tau)$ is a reflexive Banach space, $a(u, v)$ is a nondegenerate bilinear form, and $S(v)$ is linear. Moreover, by using (9),

$$
\begin{aligned}
|S(v)| & \leq \sum_{i=1}^{N}\left[\left|B_{i}\left\|Z\left(t_{i}\right)\right\| v\left(t_{i}\right)\right|\right]+\sum_{i=0}^{N} \int_{s_{i}}^{t_{i+1}}\left|A s_{i} Z(t) v(t)\right| \mathrm{d} t \\
& \leq \sum_{i=1}^{N}\left[\left|B_{i}\right|\left\|Z\left(t_{i}\right)\right\|\|v\|_{\infty}\right]+\|Z(t) A\|\|v\|_{\infty} \sum_{i=0}^{N} s_{i}\left(t_{i+1}-s_{i}\right) \\
& \leq \beta\left[\sum_{i=1}^{N}\left[\left|B_{i}\right|\left\|Z\left(t_{i}\right)\right\|\right]+\|Z(t) A\| \sum_{i=0}^{N} s_{i}\left(t_{i+1}-s_{i}\right)\right]\|v\| .
\end{aligned}
$$

Therefore, by using Theorem 1, we have the following.

Theorem 2. Noninstantaneous impulsive evolution equation (1) has a unique weak solution $u \in H_{0}^{1}(0, \tau)$ if $A$ is the generator of a uniformly continuous semigroup $\{Z(t)\}_{t \geq 0}$.

Theorem 3. Noninstantaneous impulsive evolution equation (1) has a unique weak solution $u \in H_{0}^{1}(0, \tau)$ if A generates an exponentially stable $C_{0}$-semigroup $\{Z(t)\}_{t \geq 0}$.

\section{Example of the Main Result}

Now, we present an example pertaining to the main result stated above.

Let $\quad V=L^{2}(0,1), t_{0}=s_{0}=0, t_{1}=\pi$, and $s_{1}=T=2 \pi$. Now, we let the operator $A$ be the second-order partial derivative operator, i.e., $A u=\partial^{2} u / \partial t^{2}$, for $u \in D(A)$. Then, $A$ is the infinitesimal generator of a strongly continuous semigroup of linear operators $\{Z(t)\}_{t \geq 0}$ and $Z(\cdot)$ is bounded with $\|Z(t)\| \leq e^{-t}$, for all $t \geq 0$. Now, we look at the following periodic problem: 


$$
\left\{\begin{array}{l}
\frac{\partial u(t, y)}{\partial t}=\frac{\partial^{2} u(t, y)}{\partial y^{2}}, \quad t \in(0, \pi), y \in(0,1), \\
\frac{\partial u(t, 0)}{\partial y}=\frac{\partial u(t, 1)}{\partial y}=0, \quad t \in[0, \pi], \\
u\left(\pi^{+}, y\right)=\frac{-y\left|u\left(\pi^{-}, y\right)\right|}{2\left(e^{\pi}+|u(\pi-, y)|\right)}+\left|u\left(\pi^{-}, y\right)\right|, \\
u(t)=\frac{y \cos (t)\left|u\left(\pi^{-}, y\right)\right|}{2\left(e^{\pi}+\left|u\left(\pi^{-}, y\right)\right|\right)}+\left|u\left(\pi^{-}, y\right)\right|, \quad t \in(\pi, 2 \pi] \\
u(0, y)=u(2 \pi, y) .
\end{array}\right.
$$

Let us denote $B_{i}=y \cos (t) / 2\left(e^{\pi}+\left|u\left(\pi^{-}, y\right)\right|\right)$, where $B_{i} \in B(V)$, the jump operator, and if $I \in B(V)$ is the identity operator, then $I\left(u\left(\pi^{-}, y\right)\right)=u\left(\pi^{-}, y\right)$. Then, the problem (24) can be written as

$$
\begin{aligned}
u^{\prime}(t) & =A u(t, y), \quad t \in(0, \pi), \\
u\left(\pi^{+}\right) & =\left(I+B_{i}\right)\left|u\left(\pi^{-}, y\right)\right|, \\
u(t) & =\left(I+B_{i}\right)\left|u\left(\pi^{-}, y\right)\right|, \quad t \in(\pi, 2 \pi], \\
u(0) & =u(2 \pi) .
\end{aligned}
$$

This problem has the variational form given by

$$
\int_{0}^{2 \pi} \frac{\partial u(t, y)}{\partial t} v(t) \mathrm{d} t=B_{1} Z(\pi) v(\pi)+\int_{0}^{\pi} \frac{\partial^{2} u(t, y)}{\partial y^{2}} v(t) \mathrm{d} t
$$

Since the right-hand side is bounded above and $A$ is the generator of the uniformly continuous semigroup, so, by (8), it has a unique weak solution $u \in H_{0}^{1}(0,2 \pi)$.

\section{Data Availability}

No data were used to support this study.

\section{Conflicts of Interest}

The authors declare that they have no conflicts of interest.

\section{References}

[1] H. Amann and G. Lumer, Functional Analysis and Evolution Equations, Birkhauser, Basel, Switzerland, 2008.

[2] Y. Guo, X.-B. Shu, Y. Li, and F. Xu, "The existence and Hyers-Ulam stability of solution for an impulsive Riemann-Liouville fractional neutral functional stochastic differential equation with infinite delay of order $1<\beta<2$," Boundary Value Problems, vol. 2019, no. 1, 2019.

[3] C. J. K. Batty, "One-parameter semigroups of positive operators," Bulletin of the London Mathematical Society, Lecture notes in mathematics 1184, vol. 19, no. 3, pp. 288-289, 1987.

[4] Y. Guo, M. Chen, X.-B. Shu, and F. Xu, "The existence and Hyers-Ulam stability of solution for almost periodical fractional stochastic differential equation with $\mathrm{fBm}$," Stochastic Analysis And Applications, vol. 39, no. 4, pp. 643-666, 2020.

[5] S. Li, L. Shu, X.-B. Shu, and F. Xu, "Existence and Hyers-Ulam stability of random impulsive stochastic functional differential equations with finite delays," Stochastics, vol. 91, no. 6, pp. 857-872, 2018.

[6] S. B. Agase, "Existence and stability of ordinary differential equations in locally convex spaces," Nonlinear Analysis: Theory, Methods \& Applications, vol. 5, no. 7, pp. 713-719, 1981.

[7] R. Agarwal, S. Hristova, and D. O'Regan, Non-Instantaneous Impulses in Differential Equations, p. 251, Springer International Publishing, Cham, Basel, Switzerland, 2017.

[8] L. Bai and J. Nieto, "On a delayed epidemic model with noninstantaneous impulses," Communications on Pure and Applied Analysis, vol. 19, no. 4, pp. 1915-1930, 2020.

[9] E. Hernndez, "Abstract impulsive differential equations without predefined time impulses," Journal of Mathematical Analysis and Applications, vol. 491, no. 1, Article ID 124288, 2020.

[10] E. Hernández and D. O’Regan, "On a new class of abstract impulsive differential equations," Proceedings of the American Mathematical Society, vol. 141, no. 5, pp. 1641-1649, 2012.

[11] J. Wang, "Stability of noninstantaneous impulsive evolution equations," Applied Mathematics Letters, vol. 73, pp. 157-162, 2017.

[12] Q. Tang and J. J. Nieto, "Variational approach to impulsive evolution equations," Applied Mathematics Letters, vol. 36, pp. 31-35, 2014.

[13] D. Drivaliaris and N. Yannakakis, "Generalizations of the laxmilgram theorem," Boundary Value Problems, vol. 2007, pp. 1-9, 2007.

[14] T. Hayden, "The extension of bilinear functionals," Pacific Journal of Mathematics, vol. 22, no. 1, pp. 99-108, 1967.

[15] L. Bai and J. J. Nieto, "Variational approach to differential equations with not instantaneous impulses," Applied Mathematics Letters, vol. 73, pp. 44-48, 2017.

[16] J. J. Nieto and D. O’Regan, "Variational approach to impulsive differential equations," Nonlinear Analysis: Real World Applications, vol. 10, no. 2, pp. 680-690, 2009.

[17] A. McBride, Semigroups of Linear Operators, Longman Scientific and Technical, Harlow, Essex, England, 1987. 